\title{
Spéciation de l'arsenic dans la salive et l'uri- ne humaines après exposition professionnelle
}

\section{Arsenic speciation in human saliva and urine after occupational exposure}

François BARUTHIO*, Benoît RIEGER, Patrick BIETTE, Francis PIERRE

Surveillance Biologique, INRS, Centre de Recherche - BP 27 - 54500 VANDEEUVRE - FRANCE

* Auteur à qui adresser la correspondance : François BARUTHIO, Surveillance Biologique, INRS, Centre de Recherche - BP 27 - 54500 VANDGEUVRE - FRANCE - Tél : 0383502148 - Fax : 0383502096

(Reçu le 10 mai 2001 ; accepté le 20 juin 2001)

\section{RÉSUMÉ}

La surveillance biologique des personnes exposées professionnellement aux composés de l'arsenic (As) est basée sur la mesure de la concentration urinaire de cet élément. Toutefois, l'utilisation de l'arsenic urinaire comme indicateur biologique d'exposition, nécessite que soit faite la distinction entre, d'une part les composés organiques, non toxiques, d'origine alimentaire, tels que l'arsénobétaïne et l'arsénocholine et d'autre part, les formes inorganiques et organiques toxiques, à savoir, arsénites $\left(A s^{3+}\right)$, arsénates $\left(A s^{5+}\right)$, monométhylarsonate (MMA) et diméthylarsinate (DMA), d'origine iatrogène, professionnelle ou environnementale. La pratique actuelle consiste à distinguer les composés d'origine alimentaire ou non alimentaire.

La méthode élaborée a pour objectif d'individualiser, à des fins de recherche, les composés inorganiques $\left(A s^{3+}, A s^{5+}\right)$, les métabolites organiques (MMA, DMA) et les composés d'origine alimentaire (arsénobétaïne, arsénocholine). Cela a été réalisé par chromatographie d'échange d'ions pour l'urine et la salive, en combinant deux résines, une cationique et une anionique. La méthode est directe, sans prétraitement pour les échantillons d'urine et de salive. Quatre élutions successives permettent d'individualiser 5 fractions, $\mathrm{As}^{3+}, \mathrm{As}^{5+}$, MMA, DMA et As alimentaire.

Après séparation des différentes espèces d'arsenic, l'analyse des fractions a été réalisée par spectrophotométrie d'absorption atomique électrothermique avec correction de fond par effet Zeeman et modifieur de matrice. Les méthodes décrites ont été adaptées à l'urine et à la salive à partir d'une méthode développée par Grabinski (1981) pour la

\section{SUMMARY}

Arsenic speciation in human saliva and urine after occupational exposure

François BARUTHIO, Benô̂t RIEGER, Patrick BIETTE, Francis PIERRE

The urinary excretion of absorbed or inhaled arsenic mainly in the urine is used in the biological monitoring of occupational exposure to arsenic compounds. When assessing potential hazards of arsenic inorganic and organic chemical forms there is a need for analytical methods that are able to discriminate between the different chemical forms. Speciation of arsenic is of particular interest. The inorganic arsenic species are methylated in the biological systems. Arsenite (As III) is the most toxic form and is suspected to be a human carcinogen. Arsenate (As $V$ ) is also relatively toxic, while the methylated forms monomethylarsonic acid (MMA) and dimethylarsinic acid (DMA) are less toxic. The organic compounds such as arsenobetaine and arsenocholine, often present in seafood, are non-toxic.

Several analytical procedures for arsenic compounds have been reported. The most commonly used technique is the application of chromatography for the separation and various detection systems.

The method developped in this work aims to quantify, for research studies, the inorganic compounds $\left(A s^{3+}, A s^{5+}\right)$, the organic metabolites (MMA, DMA) and the seafood origin compounds (arsenobetaine, arsenocholine). That was carried out by ion exchange chromatography for urine and saliva, by combining cationic and anionic resins. The method is direct, without pretreatment for the urine and saliva samples. 
spéciation dans l'eau. Elles ont été validées par l'évaluation de leurs critères de qualité sur l'eau surchargée en espèces chimiques et sur l'urine et la salive humaines, natives et surchargées des mêmes espèces chimiques.

La technologie analytique utilisée, permet d'atteindre des sensibilités voisines de $9 \mu \mathrm{g} / \mathrm{l}$ pour 0,0044 unités d'absorbance.sec ${ }^{-1}$. Les limites de détection (définies à 3 écarts-types de la mesure du blanc), sont de 20,3, de 18,3, 20,4 et 13,0 $\mu g / l$, respectivement pour As total urinaire, As total salivai$r e$, As urinaire non alimentaire et As dans les fractions éluées par chromatographie. Ces limites de détection sont inférieures aux valeurs de référence moyennes, et comparées à la valeur limite biologique urinaire de $50 \mu \mathrm{g} / \mathrm{g}$ de créatinine, elles permettent le suivi biologique des personnes exposées.

\section{MOTS-CLÉS}

Arsenic, analyse, speciation, surveillance biologique, urine, salive.

\section{Introduction}

La surveillance biologique des personnes exposées professionnellement aux composés de l'arsenic (As) est basée sur la mesure de la concentration urinaire de cet élément. Mais, l'utilisation de l'arsenic urinaire comme indicateur biologique d'exposition, nécessite que soit faite la distinction entre les composés organiques, non toxiques, d'origine alimentaire, tels que l'arsénobétaïne et l'arsénocholine (1) et les formes inorganiques et organiques toxiques, à savoir, arsénites $\left(\mathrm{As}^{3+}\right)$, arsénates $\left(\mathrm{As}^{\mathrm{s}}\right)$, monométhylarsonate (MMA) et diméthylarsinate (DMA), d'origine iatrogène, professionnelle ou environnementale. Les métabolites MMA et DMA sont des produits de détoxification issus de la biotransformation de l'As trivalent (2-3).

La distinction indispensable de l'origine alimentaire ou non alimentaire des composés de l'arsenic a nécessité la séparation chimique de l'arsénobétaïne et de l'arsénocholine des autres composés non alimentaires (4-6). Plusieurs procédures analytiques ont été reportées. La plus communément utilisée pour la séparation des composés de l'arsenic est la chromatographie, l'analyse étant réalisée par divers systèmes de détection.

La séparation des composés inorganiques (arsénites $\mathrm{As}^{3+}$, et arsénates $\left.\mathrm{As}^{5+}\right)$ et de leurs métabolites organiques (MMA et DMA ) a été réalisée par chromatographie par échange d'ions après adaptation à l'urine et à la salive d'une méthode décrite pour l'analyse de l'eau (5).
Four successive elutions allow to separate 5 species, $A s^{3+}$, $A s^{5+}$, MMA, DMA and food As.

After separation of the arsenic chemical species, the analysis of the fractions is performed by electrothermal atomic absorption spectrometry with Zeeman background correction and matrix modifier. The described methods are adapted to urine and saliva from a method developed by Grabinski (1981) for As speciation in tap water. The methods are validated by evaluation of their quality criteria on water spiked with the five arsenic species at a first time and human urine and saliva, spiked with the same chemical species at a second time.

This analytical technology reaches sensitivities close to 9 $\mu \mathrm{g} / \mathrm{l}$ for 0,0044 units of absorbance per second. The detection limits (defined by 3 standard deviations of blank measurements) are 20,3, 18,3,20,4 and 13,0 $\mu \mathrm{g} / \mathrm{l}$, respectively for urinary total As, salivary total As, urinary nonfood As and As after chromatographic separation. These detection limits are lower than the average reference values. Compared to the urinary biological exposure indice value of $50 \mu \mathrm{g} / \mathrm{g}$ of creatinin, they allow the biological monitoring of the exposed persons.

\section{KEY-WORDS}

Arsenic, speciation, analysis, biological monitoring, urine, saliva

Après séparation des différentes espèces d'arsenic, l'analyse a été réalisée par spectrophotométrie d'absorption atomique électrothermique avec correction de fond par effet Zeeman. Les méthodes mises au point ou adaptées ont été validées dans une première phase par l'évaluation de leurs critères de qualité (8-10) sur un milieu synthétique, l'eau surchargée en espèces chimiques étudiées, puis sur des milieux biologiques humains, urine et salive naturelles et surchargées des mêmes espèces chimiques.

\section{Matériels et réactifs}

\section{Appareillage}

L'appareillage utilisé pour la détection de l'arsenic est de la firme PERKIN ELMER Instruments et comporte :

- un spectromètre d'absorption atomique modèle 3030 ZEEMAN

- un four graphite HGA 600

- un passeur d'échantillons AS 60

- une alimentation de lampes sans électrode EDL 2

\section{Réactifs}

Les produits chimiques sont de qualité supra pure ou à défaut de qualité pour analyse la plus pure :

Etalon d'arsenic : $\mathrm{H}_{2} \mathrm{AsO}_{4}$ à $999 \pm 2 \mathrm{mg} / \mathrm{l}$ dans $\mathrm{HNO}_{3}$ 0,5 mol/l, (réf. $119773 \mathrm{MERCK}$ ).

Triton X-100 (réf. 11869 MERCK).

Nitrate de magnésium supra-pur, $\mathrm{Mg}\left(\mathrm{NO}_{3}\right)_{2}, 6 \mathrm{H}_{2} \mathrm{O}$ 


\section{(réf. 5855 MERCK).}

Chlorure de palladium $\mathrm{PdCl}_{2}$ (réf. 807110 MERCK).

Persulfate de potassium, $\mathrm{K}_{2} \mathrm{~S}_{2} \mathrm{O}_{8}$ (réf. MERCK 5092).

Acide nitrique supra pur 65,9\% (réf. MERCK 441).

Acide chlorhydrique supra pur à $32 \%$ (réf. MERCK 319).

Acide chlorhydrique concentré à $30 \%$ (réf. MERCK 318).

Toluène, $\mathrm{C}_{6} \mathrm{H}_{5} \mathrm{CH}_{3}$ rectapur (PROLABO réf. 28.675.363)

Iodure de potassium, KI, (MERCK réf. 5043)

Dichromate de sodium, $\mathrm{Na}_{2} \mathrm{Cr}_{2} \mathrm{O} 7,2 \mathrm{H}_{2} \mathrm{O}$ (CARLO ERBA réf. 478575)

Eau ultra pure système Milli Q (Société Millipore®) resistivité de 18,2 $\mathrm{M} \Omega$

Acide trichloracétique cristallisé très pur $\mathrm{CCl}_{3} \mathrm{COOH}$ (réf. MERCK 810)

Solution d'ammoniaque, Ammonium hydroxyde $\mathrm{NH}_{4} \mathrm{OH}, 33 \%$ (réf. JT. BAKER 6125)

Résine Dowex anionique : BIO-RAD AG 1-X8, réf. 140-1441

Résine Dowex cationique : BIO-RAD AG 50W-X8, réf. 142-1441

Arsenic trioxyde, $\mathrm{As}_{2} \mathrm{O}_{3}$ trivalent : (SIGMA, lot 129F0492 réf. A-1010)

Acide Arsénique (sel disodique) heptahydrate, $\mathrm{Na}_{2} \mathrm{HAsO}_{4}, 7 \mathrm{H}_{2} \mathrm{O}$, pentavalent (SIGMA, lot $80 \mathrm{H} 0474$ réf. A-6756);

Acide Monométhyl arsonique (MMA) $\mathrm{CH}_{3} \mathrm{AsO}(\mathrm{ONa})_{2}, 6 \mathrm{H}_{2} \mathrm{O}$ (CARLO ERBA, lot 0392F100, réf 371205)

Acide diméthyl arsinique (DMA), $\mathrm{C}_{2} \mathrm{H}_{6} \mathrm{AsNaO}_{2}, 3 \mathrm{H}_{2} \mathrm{O}$ (MERCK, réf. 103256)

Les solutions d'arsénobétaïne et d'arsénocholine utilisées sont des solutions non commercialisées, synthétisées par le laboratoire CNRS de Lyon-Solaise : arsénobétaïne, $\left.\left(\mathrm{CH}_{3}\right)_{3} \mathrm{AsCH}_{2} \mathrm{COO}\right)$ solution à $1 \mathrm{~g} / \mathrm{l}$ et arsénocholine, $\left(\mathrm{CH}_{3}\right)_{3} \mathrm{As}\left(\mathrm{CH}_{2}\right) 2 \mathrm{OHAsBr}$, solution à $1 \mathrm{~g} / \mathrm{l}$.

Arsénobétaïne, matériau de référence certifié $\mathrm{N}^{\circ} 626$ $(1031 \pm 6 \mathrm{mg} / \mathrm{kg})$, Bureau de Référence Communautaire (BCR) de la Commission Européenne (non disponible durant la réalisation de cette étude).

Filtre « MILLEX », porosité 0,22 $\mu \mathrm{m}$ (MILLIPORE réf. SLGP. R25 LS)

\section{Solution étalon d'arsenic :}

A partir de la solution commerciale $\mathrm{H}_{2} \mathrm{AsO}_{4}$ à $1 \mathrm{~g} / \mathrm{l}(999$ $\pm 2 \mathrm{mg} / \mathrm{l})$ sont préparées une solution intermédiaire à $50000 \mu \mathrm{g} / \mathrm{l}$ dans $\mathrm{HNO}_{3}$ à $1 \%$ (renouvelée tous les 15 jours) et une solution de travail à $500 \mu \mathrm{g} / \mathrm{l}$ dans $\mathrm{HNO}_{3}$ à $1 \%$ (renouvelée tous les jours)

\section{Modifieur de matrice :}

$10 \mu 1$ d'une solution de persulfate de palladium, préparée quotidiennement, sont ajoutés in situ dans le four graphite pour toutes les analyses d'arsenic par absorption atomique décrites dans cette étude. Ce modifieur est obtenu par mélange volume à volume des 2 solutions suivantes (préparées tous les mois) :

- $\mathbf{P d C l}_{\mathbf{2}}: 150 \mathrm{mg}$ dans $1 \mathrm{ml} \mathrm{HCl}$ concentré, après dissolution ajouter $3,75 \mathrm{ml} \mathrm{HNO}_{3}$ concentré et compléter à $25 \mathrm{ml}$ avec $\mathrm{H}_{2} \mathrm{O}$ (eau et acides ultra purs)

- $\mathbf{K}_{2} \mathbf{S}_{2} \mathbf{O}_{8}$ (persulfate de potassium) : solution à $500 \mathrm{mg}$ pour $25 \mathrm{ml} \mathrm{H} \mathrm{H}_{2} \mathrm{O}$.

Mélanger vol à vol ces 2 solutions avant l'analyse journalière. Injecter $10 \mu \mathrm{l}$ dans le four après l'injection de $15 \mu$ l d'échantillon.

\section{Modes opératoires}

\section{Analyse de l'arsenic d'origine profes- sionnelle :}

La méthode d'extraction de l'As alimentaire (4) a nécessité une mise au point pour améliorer la sensibilité et l'exactitude. Il est conseillé d'utiliser des tubes, flacons et godets en polyéthylène pour éviter toute attaque des surfaces plastiques par le toluène. Il faut prendre soin de ne pas transférer du toluène dans la phase qui sera analysée sur le spectromètre d'absorption atomique, pour éviter une interférence sur le signal d'analyse. Le tube graphite standard a été remplacé par un tube à plateforme de L'Vov avec ajout de modifieur de matrice au persulfate de palladium in situ.

\section{Principe}

Les différentes formes de l'arsenic (arsénites, arsénates, MMA et DMA à l'exception de l'arsénobétaïne et de l'arsénocholine) sont réduites par l'iodure de potassium en milieu acide. Les formes réduites sont extraites dans le toluène, puis extraites par retour de la phase organique par une solution aqueuse de dichromate de potassium.

\section{Mode opératoire}

Dans un tube en verre à bouchon vissant mélanger $1 \mathrm{ml}$ d'urine, $5 \mathrm{ml} \mathrm{HCl}$ concentré et $2 \mathrm{ml}$ d'iodure de potassium. Agiter légèrement et laisser reposer 2 minutes. Ajouter $10 \mathrm{ml}$ de toluène et agiter 15 minutes sur agitateur mécanique. Prélever et transférer $2 \mathrm{ml}$ de la phase organique dans un tube en verre en ajoutant $1 \mathrm{ml}$ de dichromate de potassium en solution $0,001 \mathrm{M}$ dans l'eau ultra pure. Agiter pendant 30 secondes au vortex (faire quelques arrêts brefs toutes les 5 secondes). Prélever la phase aqueuse avec une seringue équipée d'un filtre de type "Millex" pour éliminer le toluène. Transférer cette phase aqueuse dans un tube conique en polyéthylène. 


\section{Analyse}

La phase aqueuse filtrée est analysée par spectrométrie d'absorption atomique électrothermique. L'étalonnage du spectromètre est réalisé par ajouts dosés dans un extrait d'urine exempt d'arsenic (pool d'urine "normale" à concentration d'arsenic non détectable) : Tableau 1.

\section{Paramètres instrumentaux :}

\begin{tabular}{|c|c|}
\hline Signal & superficie de pic \\
\hline Étalonnage & linéaire \\
\hline Lampe EDL 2 & $400 \mathrm{~mA}$ \\
\hline Longueur d'onde & $193,7 \mathrm{~nm}$ \\
\hline Bande passante & $0,7 \mathrm{~nm}$ \\
\hline Four graphite & $\begin{array}{l}\text { le tube standard a été rem- } \\
\text { placé par le tube pyroly- } \\
\text { tique à plateforme de } \\
\text { L'Vov dont la sensibilité } \\
\text { est meilleure }\end{array}$ \\
\hline
\end{tabular}

Gaz (vecteur et protection) argon $\mathrm{U}$

Volume injecté

$15 \mu \mathrm{l}$

Modifieur de matrice

$10 \mu \mathrm{d}$ du mélange $\mathrm{PdCl}_{2}$ $/ \mathrm{K}_{2} \mathrm{~S}_{2} \mathrm{O}_{8}$

Correction de fond

effet Zeeman

Puissance maximum et Gaz-stop sont appliqués durant l'atomisation.

Programme thermique : Tableau 2.

\section{Mode opératoire}

L'extrait est transféré directement dans les godets du spectromètre $(800 \mu \mathrm{l}+200 \mu \mathrm{l}$ d'eau ultra-pure). Cette dilution au $4 / 5$ permet de préparer une gamme de "calibration par ajouts dosés". Eviter d'entraîner du toluène qui pourrait perturber le signal.

$800 \mu \mathrm{l}$ d'extrait contiennent $800 / 5=160 \mu \mathrm{l}$ d'urine non diluée, soit $160 \mu \mathrm{l}$ d'urine dans $1000 \mu \mathrm{l}$ du godet, dans les $15 \mu \mathrm{l}$ injectés il y a $160 / 1000 * 15=2,4 \mu \mathrm{l}$ d'urine non diluée.

Les critères de qualité de cette méthode sont résumés dans le tableau 1 . Les résultats du contrôle de qualité externe du Centre de Toxicologie du Québec (CTQ) montrent une corrélation satisfaisante avec les valeurs cibles (figure 1).

Les résultats du contrôle de qualité interne (urines «Lyphochek $\left.{ }^{\oplus} »\right)$ sont reportés dans le tableau 1. Les moyennes des mesures (avec \pm 1 écart-type) sont proches de la valeur cible.

Comme pour l'arsenic total nous avons réalisé une intercomparaison avec un laboratoire utilisant la méthode directe des hydrures (11). La corrélation entre les deux méthodes est très satisfaisante :

$\mathrm{r}=0,9984 \quad(\mathrm{n}=10) \quad \mathrm{p}<5.10^{-6}$

\section{Analyse de l'arsenic total dans la salive et dans l'urine}

\section{Paramètres instrumentaux}

Les paramètres instrumentaux suivants sont appliqués :

Lampe sans électrode $\quad 400 \mathrm{~mA}$

Longueur d'onde $\quad 193,7 \mathrm{~nm}$

Bande passante $\quad 0,7 \mathrm{~nm}$

Four graphite pyrolytique à platforme de

L'Vov

Gaz (vecteur et protection) argon U

Volume injecté

$15 \mu 1$ d'urine diluée au $1 / 5$ avec le diluant

Modifieur de matrice $\quad 10 \mu \mathrm{lde} \mathrm{PdCl}_{2} / \mathrm{K}_{2} \mathrm{~S}_{2} \mathrm{O}_{8}$

Correction de fond effet Zeeman

Puissance maximum et Gaz-stop sont appliqués durant l'atomisation.

Tableau I

\begin{tabular}{|cccccc|}
\hline & $\begin{array}{c}\text { Extrait } \\
\text { aqueux }\end{array}$ & $\mathbf{H}_{\mathbf{2}} \mathbf{O}$ & Ajout-500 $\boldsymbol{\mu g} / \mathbf{l}$ & $\begin{array}{c}\text { concentration } \\
\text { injectée } \\
\boldsymbol{\mu g / /}\end{array}$ & $\begin{array}{c}\text { concentration } \\
\boldsymbol{\mu g} / \mathbf{l}\end{array}$ \\
& $(\boldsymbol{\mu l})$ & $\boldsymbol{\mu l}$ & $\boldsymbol{\mu l}$ & $\begin{array}{c}\text { dans échant } \\
\text { pur }\end{array}$ \\
\hline Blanc réactifs & 800 & 200 & 0 & 0 & 0 \\
Blanc échant. & 800 & 200 & 0 & 0 & 0 \\
Standard 1 & 800 & 150 & 50 & 25 & 31,25 \\
Standard 2 & 800 & 100 & 100 & 50 & 62,5 \\
Standard 3 & 800 & 50 & 150 & 75 & 93,75 \\
Standard 4 & 800 & 0 & 200 & 100 & 125 \\
\hline Échant. inconnu & 800 & 200 & 0 & 0 & 0 \\
\hline
\end{tabular}


Tableau II

\begin{tabular}{|lcccc|}
\hline & Température $\left({ }^{\circ} \mathbf{C}\right)$ & Rampe(s) & Durée(s) & Gaz argon (ml/min) \\
\hline Séchage & 90 & 3 & 10 & 300 \\
Minéralisation & 110 & 10 & 30 & 300 \\
& 300 & 10 & 25 & 300 \\
Atomisation & 1350 & 10 & 30 & 300 \\
Pyrolyse & 2400 & 0 & 3 & 0 \\
& 2600 & 1 & 3 & 300 \\
& 20 & 1 & 3 & 300 \\
\hline
\end{tabular}

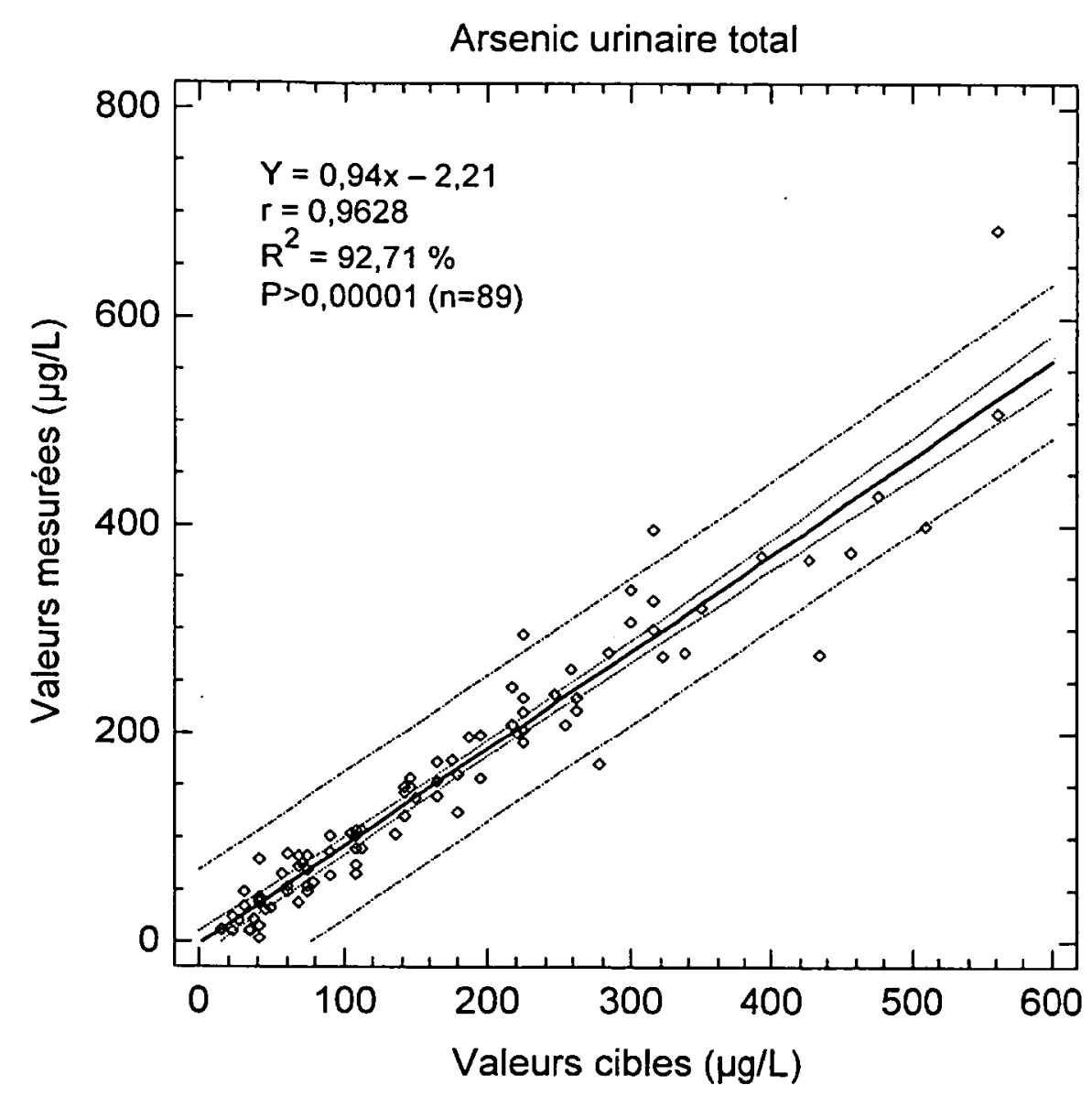

Figure 1 : Corrélation valeurs mesurées/valeurs cibles pour le contrôle de qualité externe pour l'analyse de l'arsenic urinaire total.

Diluant : $2 \mathrm{~g}$ de Triton $\mathrm{X}-100^{\circledast}$ et $1,4 \mathrm{~g}$ de $\left(\mathrm{NO}_{3}\right)_{2} \mathrm{Mg}$ $6 \mathrm{H}_{2} \mathrm{O}$ pour 1 litre

\section{Analyse directe de l'arsenic total dans la salive}

L'étalonnage du spectromètre est réalisé par ajouts dosés dans un pool de salive « normale » à concentration d'arsenic non détectable, (Tableau 3).

\section{Analyse de l'arsenic total dans l'urine}

L'étalonnage est effectué par ajouts dosés dans un pool d'urine à concentration d'arsenic non detectable, (Tableau 4).

\section{Programme thermique du spectromètre d'absorption atomique $3030 \mathrm{Z}$ pour}

l'analyse de l'arsenic total dans l'urine et la salive : (Tableau 5).

Les critères de qualité de ces méthodes sont résumés dans le tableau 1. Les résultats du contrôle de qualité externe (CTQ) présentent une corrélation satisfaisante avec les valeurs cibles (figure 2).

Les résultats du contrôle de qualité interne (urines «Lyphochek ${ }^{{ }}$) ) sont reportés dans le tableau 1. Les moyennes des mesures (avec \pm 1 écart-type) sont proches de la valeur cible.

Nous avons réalisé une intercomparaison avec un laboratoire utilisant la méthode directe des hydrures (11). Les résultats obtenus avec l'urine indiquent une bonne corrélation entre les deux méthodes :

$$
\mathrm{r}=0,8963 \quad(\mathrm{n}=10) \quad \mathrm{p}>5.10^{-4}
$$

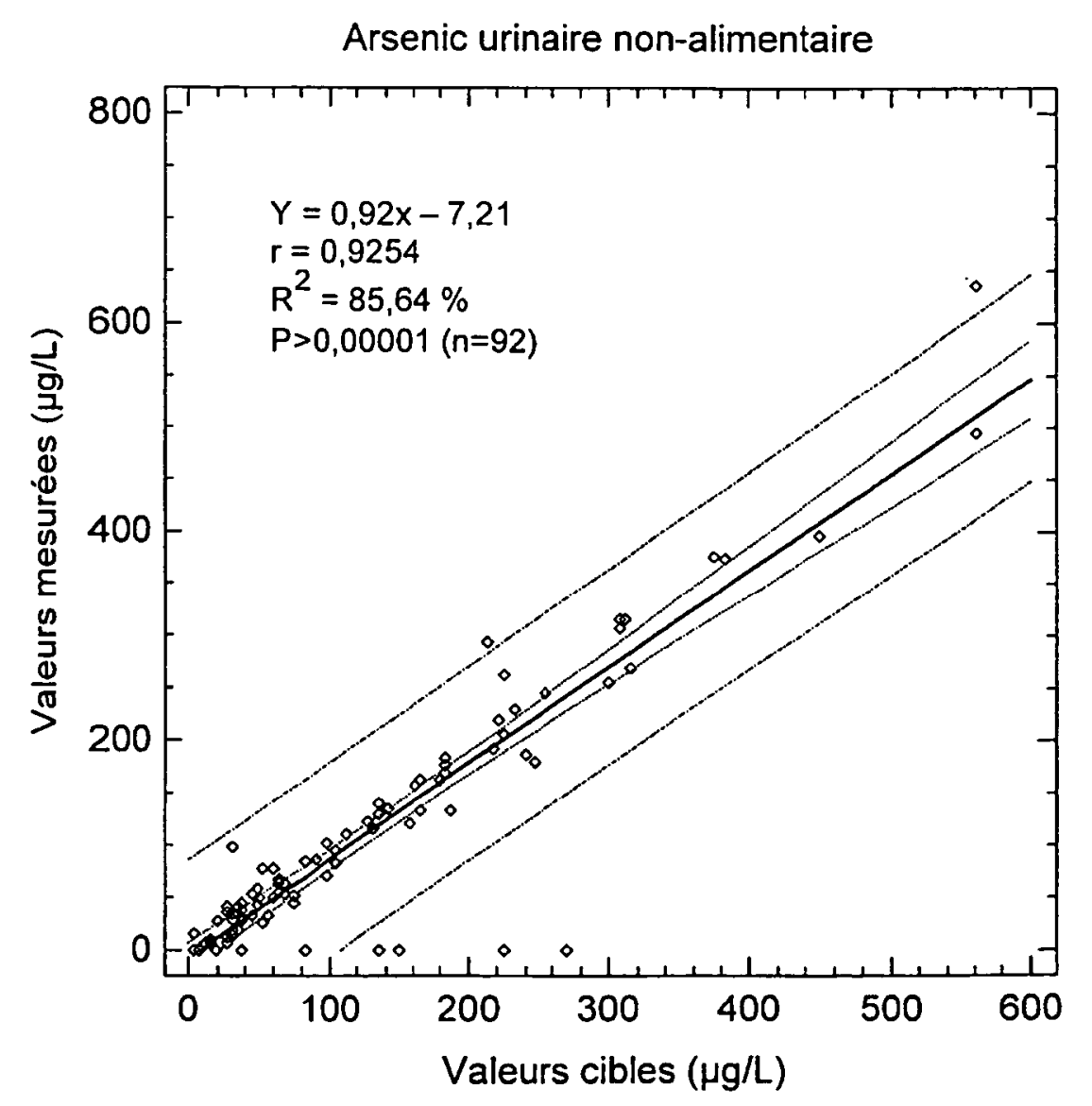

Figure 2 : Corrélation valeurs mesurées/valeurs cibles pour le contrôle de qualité externe pour l'analyse de l'arsenic urinaire non alimentaire d'origine professionnelle. 
Annales de Toxicologie Analytique, vol. XIII, n³, 2001

Tableau III

\begin{tabular}{|ccccccc|}
\hline & Salive & Diluant & $\mathbf{H}_{\mathbf{2}} \mathbf{O}$ & $\begin{array}{c}\text { Ajout à } \\
\mathbf{5 0 0} \boldsymbol{\mu g} / \mathbf{l} \\
\boldsymbol{\mu l}\end{array}$ & $\begin{array}{c}\text { Conc. } \\
\text { injectée } \\
\boldsymbol{\mu g} / \mathbf{l}\end{array}$ & $\begin{array}{c}\text { Conc. } \\
\boldsymbol{\mu g} / \mathbf{l} \\
\text { (échant) }\end{array}$ \\
\hline $\begin{array}{c}\text { Blanc réactifs } \\
\text { Blanc échant. }\end{array}$ & 0 & $\boldsymbol{\mu l}$ & $\boldsymbol{\mu l}$ & 0 & 0 & 0 \\
(pool salive) & 200 & 600 & 200 & 0 & 0 & 0 \\
Ajout 1 & 200 & 600 & 150 & 50 & 25 & 125 \\
Ajout 2 & 200 & 600 & 100 & 100 & 50 & 250 \\
Ajout 3 & 200 & 600 & 50 & 150 & 75 & 375 \\
Ajout 4 & 200 & 600 & 0 & 200 & 100 & 500 \\
Échantillon X & 200 & 600 & 200 & 0 & $x$ & x \\
\hline
\end{tabular}

Tableau IV

\begin{tabular}{|c|c|c|c|c|c|c|}
\hline & $\begin{array}{c}\text { Urine } \\
\mu l\end{array}$ & $\begin{array}{c}\text { Diluant } \\
\quad \mu l\end{array}$ & $\begin{array}{r}\mathbf{H}_{2} \mathrm{O} \\
\boldsymbol{\mu l}\end{array}$ & $\begin{array}{c}\text { Ajout à } \\
500 \mu \mathrm{g} / \mathrm{l} \\
\mu \mathrm{l}\end{array}$ & $\begin{array}{c}\text { Injection } \\
\mu \mathrm{g} / \mathrm{l}\end{array}$ & $\begin{array}{c}\text { Conc. } \\
\mu \mathrm{g} / \mathrm{l} \\
\text { (échantillon) }\end{array}$ \\
\hline Blanc réactifs & 0 & 600 & 400 & 0 & 0 & 0 \\
\hline Urine & 200 & 600 & 200 & 0 & 0 & 0 \\
\hline Ajout 1 & 200 & 600 & 150 & 50 & 25 & 125 \\
\hline Ajout 2 & 200 & 600 & 100 & 100 & 50 & 250 \\
\hline Ajout 3 & 200 & 600 & 50 & 150 & 75 & 375 \\
\hline Ajout 4 & 200 & 600 & 0 & 200 & 100 & 500 \\
\hline Échantillon X & 200 & 600 & 200 & 0 & $\mathrm{x}$ & $\mathrm{x}$ \\
\hline
\end{tabular}

Tableau V

\begin{tabular}{|lcccc|}
\hline & Température $\left({ }^{\circ} \mathbf{C}\right)$ & Montée(s) & Maintien(s) & Gaz argon (ml/min) \\
\hline Séchage & 110 & 10 & 35 & 300 \\
Minéralisation & 300 & 10 & 30 & 300 \\
Atomisation & 1350 & 10 & 30 & 300 \\
Nettoyage & 2400 & 0 & 4 & 0 \\
& 2600 & 1 & 3 & 300 \\
& 20 & 1 & 3 & 300 \\
\hline
\end{tabular}




\section{Spéciation des composés de l'arsenic dans la salive et l'urine :}

\section{Séparation des espèces As par chromato- graphie par échange d'ions :}

La technique de séparation utilisée dérive de celle décrite pour l'eau par Grabinski (5).

Des mélanges des cinq espèces chimiques disponibles ont été réalisés dans l'eau, la salive et l'urine avec le trioxyde de diarsenic (As3+), l'arsénate de sodium (As $5+$ ), le monométhylarsonate de sodium (MMA), l'acide diméthylarsinique (DMA) et l'arsénobetaïne à raison de $500 \mathrm{ng}$ de chaque dans $2 \mathrm{ml}$ d'eau, de pool de salive ou de pool d'urine.

Les colonnes en verre de $1 \mathrm{~cm}$ de diamètre et $50 \mathrm{~cm}$ de longueur ont été remplies avec une résine anionique (BIO-RAD AG 1-X8) sur une hauteur de $10 \mathrm{~cm}$. La seconde résine, cationique (BIO-RAD AG 50W-X8) sur une hauteur de $25 \mathrm{~cm}$ est déposée sur la résine anionique, en prenant soin de ne pas mélanger les 2 résines à l'interface (figure 3).

Les colonnes neuves doivent être saturées en arsenic par dépôt de $50 \mu \mathrm{g}$ de chaque espèce, puis rincées, avant leur première utilisation. Le rinçage, comme la régénération des colonnes après chaque séparation est réalisé en passant en séquence, plusieurs fois après saturation des colonnes neuves et 2 fois pour régénérer les colonnes, $70 \mathrm{ml}$ des 3 solutions suivantes : $\mathrm{NH}_{4} \mathrm{OH}$ $1,5 \mathrm{M}$ puis $\mathrm{HCl} 1 \mathrm{M}$ et finalement $\mathrm{HCl} 0,48 \mathrm{M}$.

Quatre éluants successifs sont nécessaires pour une séparation complète des 5 composés : $55 \mathrm{ml}$ d'acide trichloracétique à $0,006 \mathrm{M}$ puis $8 \mathrm{ml}$ d'acide trichloracé-

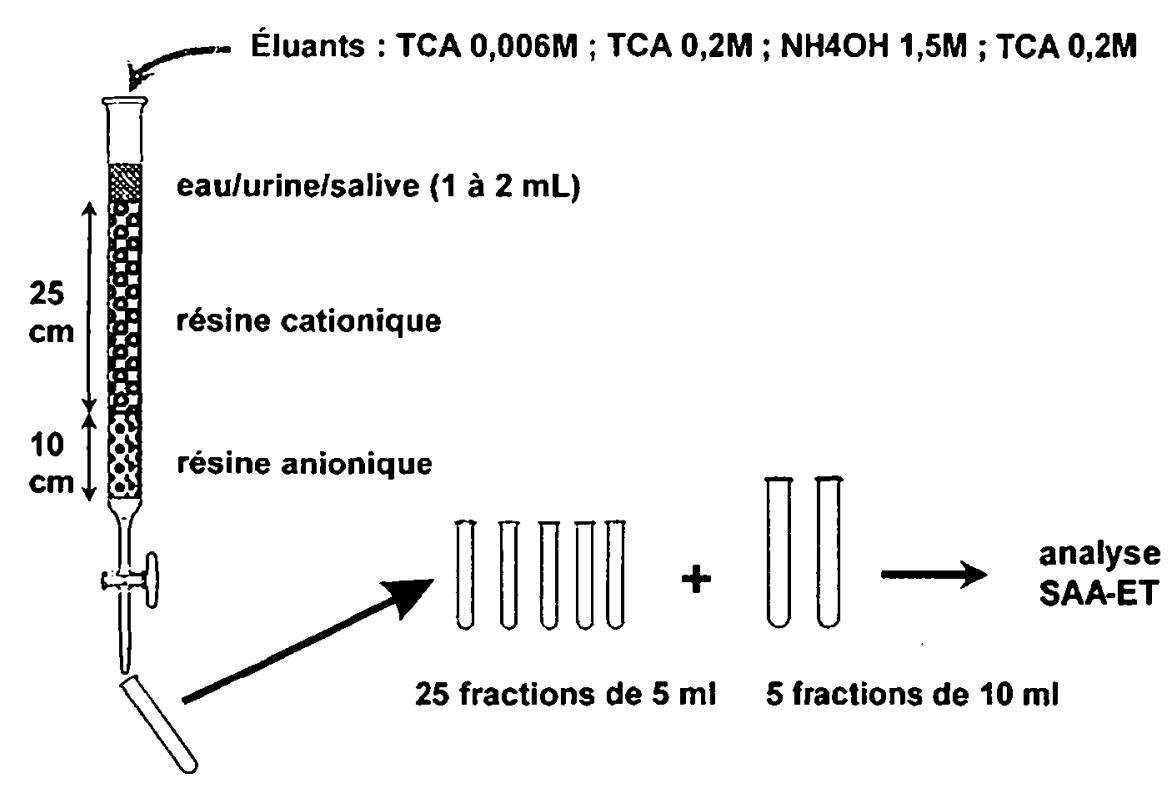

Figure 3 : Schéma de la séparation chromatographique par échange d'ions. tique à $0,2 \mathrm{M}, 55 \mathrm{ml}$ d'hydroxyde d'ammonium à 1,5 $\mathrm{M}$ et finalement $50 \mathrm{ml}$ d'acide trichloracétique à $0,2 \mathrm{M}$ (figure 3 ). Chacune des espèces a d'abord été éluée en solution pure $(1 \mathrm{ml}$ à $10 \mu \mathrm{g} / \mathrm{ml})$ puis en mélange. Ceci nous a permis d'identifier chacun des composés du mélange sur les chromatogrammes, (figure 4 pour l'eau et la salive et figures 5 et 6 pour l'eau et l'urine). Pour les surcharges dans les trois milieux, eau, salive et urine, mélanger $50 \mu \mathrm{l}$ à $10 \mu \mathrm{g} / \mathrm{ml}(0,5 \mu \mathrm{g})$ de chaque espèce chimique et compléter à $2 \mathrm{ml}$ avec chacun des 3 milieux étudiés.

\section{Analyse de l'arsenic dans les fractions éluées :}

L'analyse de l'arsenic dans les fractions éluées a été réalisée par spectrométrie d'absorption atomique électrothermique à correction de fond par effet Zeeman. $15 \mu \mathrm{l}$ des fractions éluées sont injectés dans le four graphite sans dilution. $10 \mu \mathrm{l}$ de modifieur de matrice (persulfate de palladium) sont injectés à la suite de l'échantillon dans le four graphite.

\section{Étalonnage :}

La gamme d'étalonnage est réalisée dans l'acide trichloracétique 0,2M, (Tableau 6).

Les fractions éluées sur les colonnes sont injectées dans le four graphite sans dilution

\section{Programme thermique : (Tableau 7).}

La chromatographie par échange d'ions a permis une excellente séparation des 5 espèces de l'arsenic, inorganiques $\left(\mathrm{As}^{3+}, \mathrm{As}^{5+}\right)$, organiques ( MMA et DMA ) et de l'arsenic d'origine alimentaire.

La séparation des 5 espèces de l'arsenic est représentée en annexe 2 pour l'eau et la salive sur la figure 4 , pour l'eau et l'urine sur la figure 5 , pour l'urine après exposition professionnelle et l'urine après consommation de poissons et de fruits de mer sur la figure 6 .

Les rendements de la séparation chromatographique (pourcentages de l'arsenic total élué par rapport à l'arsenic total déposé sur les colonnes) sont de 90 à $98 \%$ pour l'eau, de 84 à $95 \%$ pour l'urine, et de 84 à $90 \%$ pour la salive.

\section{Discussion et conclusions}

La technologie analytique utilisée dans cette étude (SAA-ET), permet d'atteindre des sensibilités et des limites de détection très satisfaisantes. Ces limites de détection sont inférieures aux valeurs de référence moyennes qui varient entre 15 et $70 \mu \mathrm{g} / \mathrm{l}$ selon les pays, les professions ou l'environnement, et comparées à la valeur limite biologique de $50 \mu \mathrm{g} / \mathrm{g}$ de créatinine (12), elles permettent le suivi biologique des personnes exposées. 


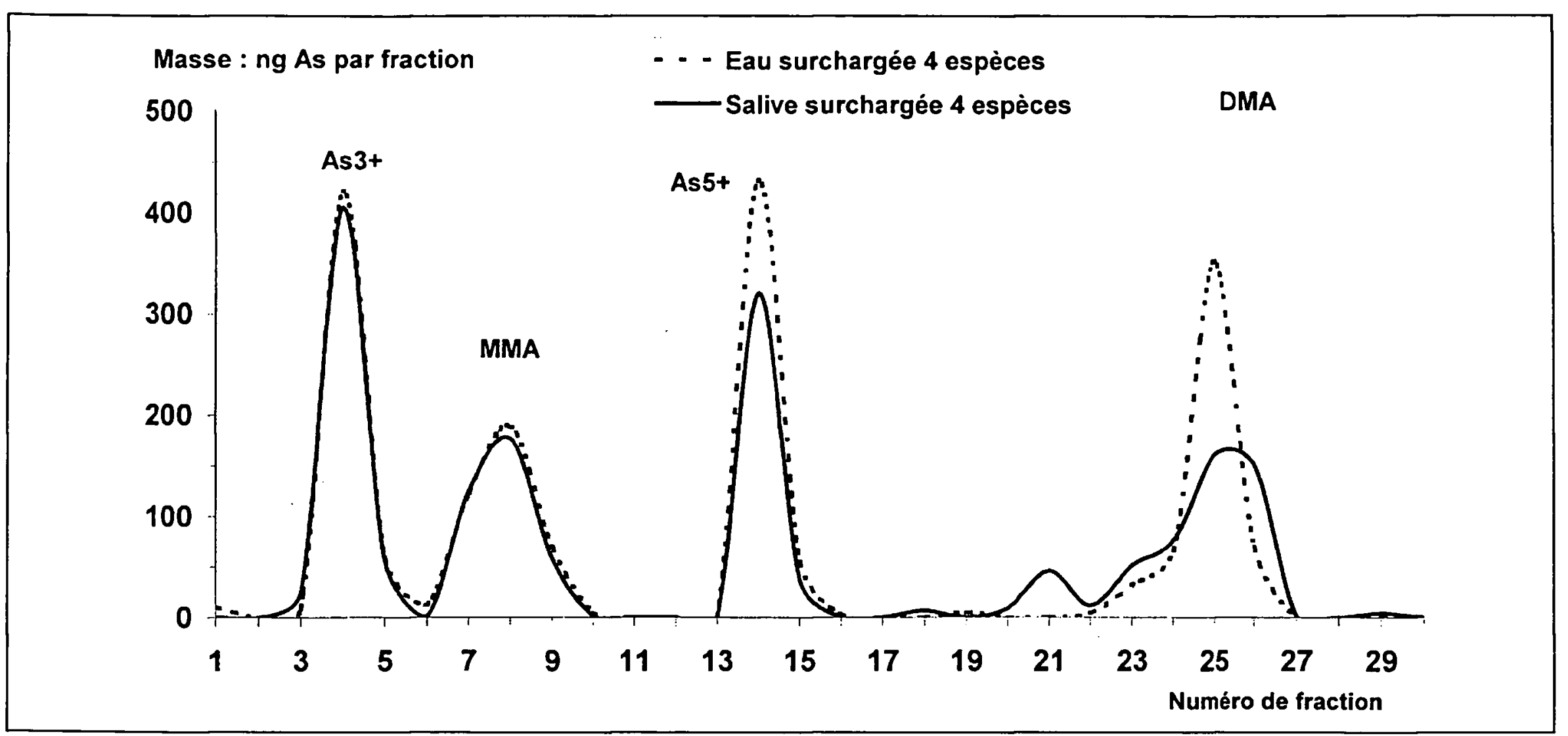

Figure 4 : Séparation comparée des 5 espèces dans l'eau et la salive.

Tableau VI

\begin{tabular}{|ccccc|}
\hline & T.C.A. $\mathbf{0 , 2 M}$ & $\mathbf{H}_{\mathbf{2}} \mathbf{O}$ & Ajout à $\mathbf{5 0 0} \boldsymbol{\mu g} / \mathbf{l}$ & $\begin{array}{c}\text { concentration } \\
\text { ajouté } \boldsymbol{\mu g} / \mathbf{l}\end{array}$ \\
& $\boldsymbol{\mu l}$ & $\boldsymbol{\mu l}$ & (ds diluant) $\boldsymbol{\mu l}$ & (échant pur) \\
\hline Blanc (TCA 0,2 M) & 800 & 200 & 0 & 0 \\
Ajout 1 & 800 & 150 & 50 & 25 \\
Ajout 2 & 800 & 100 & 100 & 50 \\
Ajout 3 & 800 & 50 & 150 & 75 \\
Ajout 4 & 800 & 0 & 200 & 100 \\
\hline
\end{tabular}

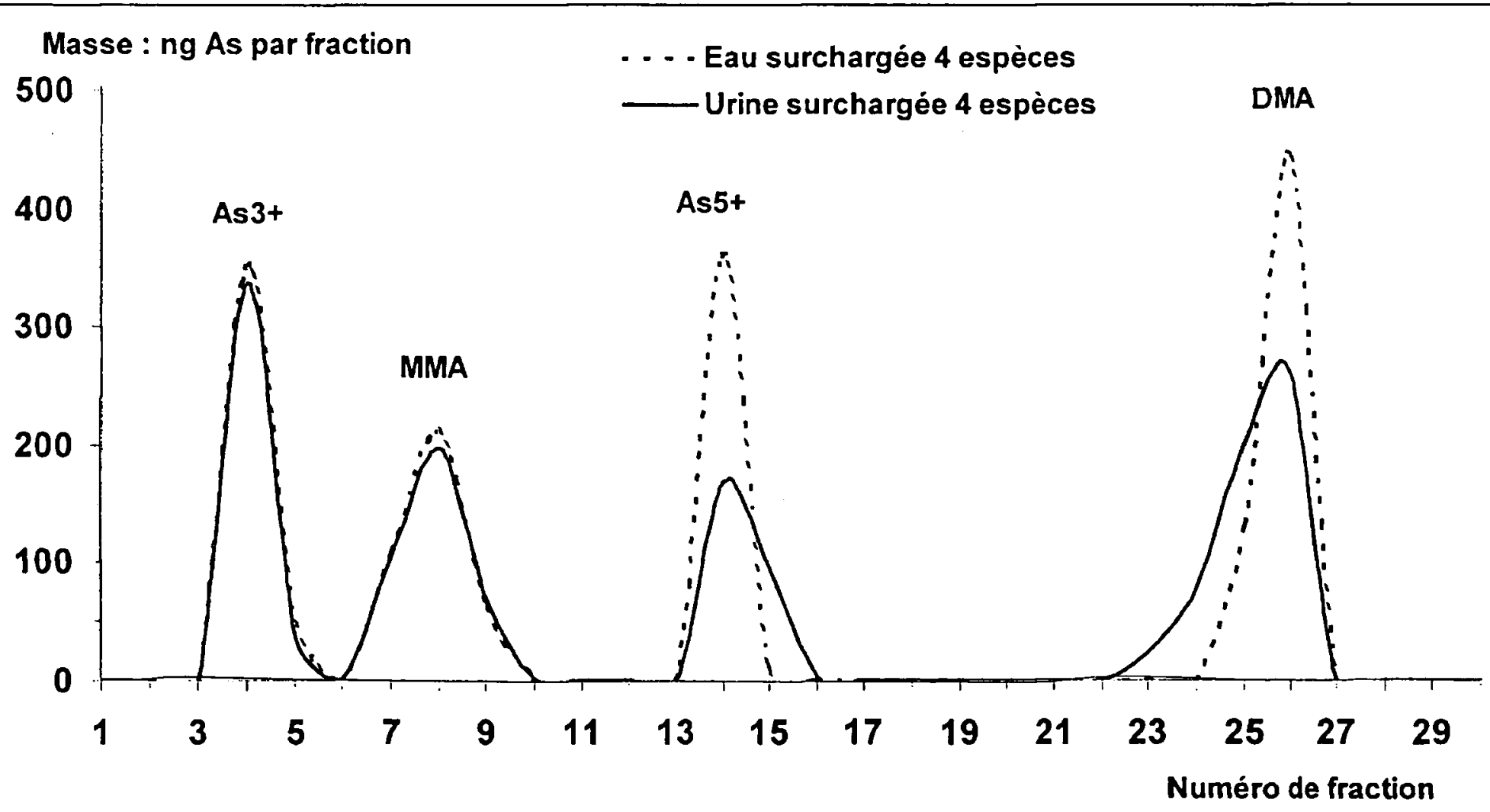

Figure 5 : Séparation comparée des 5 espèces dans l'eau et l'urine. 
Tableau VII

\begin{tabular}{|lcccc|}
\hline & Température $\left({ }^{\circ} \mathbf{C}\right)$ & Montée(s) & Maintien(s) & Gaz argon $(\mathbf{m l} / \mathbf{m i n})$ \\
\hline Séchage & 90 & 6 & 35 & 300 \\
Minéralisation & 250 & 5 & 15 & 300 \\
& 700 & 10 & 20 & 300 \\
Atomisation & 1350 & 1 & 20 & 300 \\
Nettoyage & 2400 & 0 & 3 & 0 \\
& 2700 & 1 & 3 & 300 \\
& 20 & 1 & 3 & 300 \\
\hline
\end{tabular}

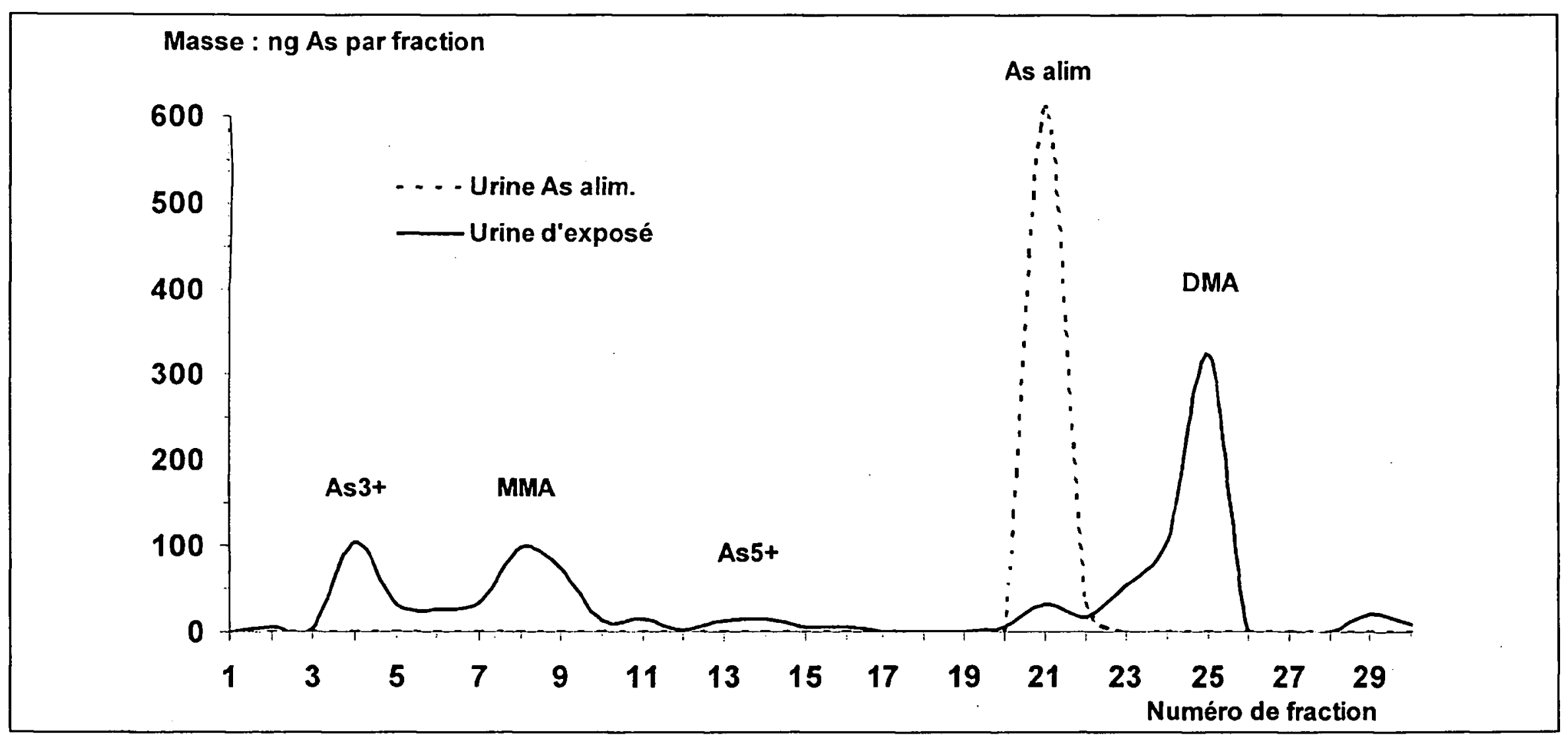

Figure 6 : Séparation comparée des 5 espèces dans l'urine d'un ouvrier exposé et As d'origine alimentaire dans l'urine d'un volontaire.

\section{Références}

1. Arbouine M.W., Wilson H.K. - The effect of seafood consumption on the assessment of occupational exposure to arsenic by urinary arsenic speciation measurements. Journal of Trace Elements and Electrolytes in Health and Disease, 1992, 6, pp. 153-160.

2. Buratti M, Calzaferri G, Caravelli G, Colombi A, Maroni M. - Significance of arsenic metabolic forms in urine.Part I.Chemical speciation. International Journal of Environmental Analytical Chemistry, 1984, 17, pp. 25-34.

3. Farmer J.G., Johnson L.R. - Assessment of occupational exposure to inorganic arsenic based on urinary concentrations and speciation of arsenic. British Journal of Industrial Medicine, 1990, 47, pp. 342-348.

4. Fitchett A.W., Daughtrey E.H. Jr., Mushak P. Quantitative measurements of inorganic and organic arsenic by flameless atomic absorption spectrometry. Analytica Chimica Acta, 1975, 79, pp. 93-99.
5. Grabinski A.A. - Determination of arsenic(III), Arsenic(V), monomethylarsonate and dimethylarsinate by ion exchange chromatography with flameless atomic absorption spectrometric detection. Analytical Chemistry, 1981, 53, pp. 966-968.

6. Buchet J.P., Lauwerys R., Roels H. - Comparison of several methods for the determination of arsenic compounds in water and urine.Their application for the study of arsenic metabolism and monitoring workers exposed to arsenic. International Archives Occupational and Environmental Health, 1980, 46, pp. 11-29.

7. Nixon D.E., Mussmann G.V., Eckdahl S.J., Moyer T.P. Total arsenic in urine: palladium-persulfate vs nickel as a matrix modifier for graphite furnace atomic absorption spectrophotometry. Clinical Chemistry, 1991, 37, pp. 1575-1579. 
8. Long G.L., Winefordner J.D. - Limit of detection. A closer look at the IUPAC definition. Analytical Chemistry, 1983, 55, pp. 712A-724A.

9. Vassault A., Grafmeyer D., Naudin C., Dumont G., Bailly M., Henny J., Gerhardt M.F., Georges P. - Protocol for the validation of methods. Annales de Biologie Clinique, 1986, 44, pp. 686-745.

10. Cornelis $\mathrm{R}$ - Recommendations of the international union of pure and applied chemistry concerning analytical quality criteria in the biological monitoring of toximetals. Scandinavian Journal of Work Environment \& Health, 1993,19, pp. 14-18.
11. Malikouti H, Javelaud B, Lallement B, Boudene CL. Intérêt d'une méthode de dosage de l'arsenic urinaire sans minéralisation préalable de l'échantillon. 1. L'arsenic urinaire dans la population générale et chez les mineurs de mispickel. Archives des Maladies Professionnelles, 1989, 50, pp. 79-86.

12. INRS - Indices biologiques d'exposition. Cahiers de Notes Documentaires, 1995,160, pp. 355-367.

Tableau I Annexes : Critères de qualité des méthodes d'analyse.

\begin{tabular}{|c|c|c|c|c|}
\hline Critère & As total Salive & $\begin{array}{c}\text { Arsenic total } \\
\text { Urine }\end{array}$ & $\begin{array}{c}\text { Arsenic non } \\
\text { alimentaire Urine }\end{array}$ & $\begin{array}{l}\text { As fractions } \\
\text { éluées }\end{array}$ \\
\hline $\begin{array}{l}\mathrm{LD}^{*}(\mu \mathrm{g} / \mathrm{l}) \\
\mathrm{LDa}^{* *}(\mathrm{ng})\end{array}$ & $\begin{array}{c}18,3 \mu \mathrm{g} / \mathrm{l} \\
0,08 \mathrm{ng} \\
(\mathrm{n}=27)\end{array}$ & $\begin{array}{c}20,3 \mu \mathrm{g} / 1 \\
0,11 \mathrm{ng} \\
(\mathrm{n}=31)\end{array}$ & $\begin{array}{c}20,4 \mu \mathrm{g} / 1 \\
0,06 \mathrm{ng} \\
(\mathrm{n}=27)\end{array}$ & $\begin{array}{c}13,0 \mu \mathrm{g} / \mathrm{l} \\
0,14 \mathrm{ng} \\
(\mathrm{n}=38)\end{array}$ \\
\hline $\begin{array}{l}\text { Exactitude : } \\
\text { CQ externe } \\
\text { du CTQ }\end{array}$ & $\begin{array}{l}\text { Absence de } \\
\text { matériaux de } \\
\text { référence }\end{array}$ & $\begin{array}{c}y=0,9327 x+1,564 \\
r=0,9661 n=23 \\
p<0,000005\end{array}$ & $\begin{array}{c}y=0,9231 x-9,433 \\
r=0,9891 n=23 \\
p<0,000005\end{array}$ & $\begin{array}{c}\text { Programme de } \\
\text { comparaisons } \\
\text { interlabo } \\
\text { inexistant }\end{array}$ \\
\hline $\begin{array}{c}\text { Exactitude : } \\
\mu \mathrm{g} / \mathrm{l} \\
\text { CQ interne } \\
\text { "Lyphocheck®" }\end{array}$ & $\begin{array}{l}\text { Absence de } \\
\text { matériaux de } \\
\text { référence }\end{array}$ & $\begin{array}{c}59,4 \pm 7,4(\mathrm{n}=40) \\
\text { cible }: 58(47-70) \\
\\
160,8 \pm 13,8(\mathrm{n}=42) \\
\text { cible }: 148(118-177)\end{array}$ & $\begin{array}{c}48,2 \pm 9,17(\mathrm{n}=23) \\
\text { cible }: 58(47-70) \\
\\
132,3 \pm 14,0(\mathrm{n}=23) \\
\text { cible }: 148(118-177)\end{array}$ & $\begin{array}{l}\text { Absence de } \\
\text { matériaux de } \\
\text { référence }\end{array}$ \\
\hline Répétabilité & $\begin{array}{c}6,6 \% \text { à } 125 \mu \mathrm{g} / \mathrm{l} \\
\mathrm{n}=20 \\
3,5 \% \text { à } 500 \mu \mathrm{g} / \mathrm{l} \\
\mathrm{n}=20\end{array}$ & $\begin{array}{c}5 \% \text { à } 125 \mu \mathrm{g} / \mathrm{l} \\
\mathrm{n}=15 \\
3 \% \text { à } 500 \mu \mathrm{g} / \mathrm{l} \\
\mathrm{n}=20\end{array}$ & $\begin{array}{c}4,6 \% \text { à } 23 \mu \mathrm{g} / \mathrm{l} \\
\mathrm{n}=48 \\
1,8 \% \text { à } 104 \mu \mathrm{g} / 1 \\
\mathrm{n}=31\end{array}$ & $\begin{array}{c}9,9 \% \text { à } 15 \mu \mathrm{g} / \mathrm{l} \\
\mathrm{n}=39 \\
3,2 \% \text { à } 111 \mu \mathrm{g} / \mathrm{l} \\
\mathrm{n}=40\end{array}$ \\
\hline Reproductibilité & $\begin{array}{c}5,6 \% \text { à } 125 \mu \mathrm{g} / \mathrm{l} \\
\mathrm{n}=26 \\
2,3 \% \text { à } 375 \mu \mathrm{g} / \mathrm{l} \\
\mathrm{n}=22\end{array}$ & $\begin{array}{c}8,6 \% \text { à } 125 \mu \mathrm{g} / \mathrm{l} \\
\mathrm{n}=31 \\
5,2 \% \text { à } 250 \mu \mathrm{g} / \mathrm{l} \\
\mathrm{n}=31\end{array}$ & $\begin{array}{c}5,6 \% \text { à } 31,25 \mu \mathrm{g} / \mathrm{l} \\
\mathrm{n}=22 \\
2,7 \% \text { à } 62,5 \mu \mathrm{g} / 1 \\
\mathrm{n}=22\end{array}$ & $\begin{array}{c}3 \% \text { à } 100 \mu \mathrm{g} / \mathrm{l} \\
\mathrm{n}=27 \\
5,9 \% \text { à } 50 \mu \mathrm{g} / \mathrm{l} \\
\mathrm{n}=27\end{array}$ \\
\hline $\begin{array}{c}\text { Sensibilité } \\
\mu \mathrm{g} / \mathrm{l} / 0,0044 \\
\text { abs-S }\end{array}$ & 8,10 & 9,23 & 9,55 & 2,56 \\
\hline $\begin{array}{l}\text { Droite de } \\
\text { calibration } \\
\text { moyenne }\end{array}$ & $\begin{array}{c}\mathrm{y}=0,000544 \mathrm{x}-9,31 \cdot 10^{-8} \\
\mathrm{r}=0,9996 \quad \mathrm{n}=7\end{array}$ & $\begin{array}{c}y=0,00045 x-0,0002 \\
r=1,0000 \quad n=31\end{array}$ & $\begin{array}{c}y=0,0021 x-4,3 \cdot 10^{-7} \\
r=0,9998 \quad n=22\end{array}$ & $\begin{array}{c}y=0,00172 x-2,93 \cdot 10^{-6} \\
r=0,99998 \quad n=27\end{array}$ \\
\hline Linéarité & jusqu'à $500 \mu \mathrm{g} / \mathrm{l}$ & jusqu'à $500 \mu \mathrm{g} / \mathrm{l}$ & jusqu'à $625 \mu \mathrm{g} / 1$ & jusqu'à $250 \mu \mathrm{g} / \mathrm{l}$ \\
\hline
\end{tabular}

${ }^{*}$ LD (Limite de détection) $=3$ sd (3 écarts-types de la mesure du blanc)

**LDa (Limite de détection absolue) $=3$ sd, exprimée en ng par prise d'essai 